\title{
Supplement to "Asymptotically efficient estimation of a scale parameter in Gaussian time series and closed-form expressions for the Fisher information"
}

\section{TILL SABEL ${ }^{1}$ and JOHANNES SCHMIDT-HIEBER ${ }^{2}$}

${ }^{1}$ Institut für Mathematische Stochastik, Universität Göttingen, Goldschmidtstr. 7, 37077 Göttingen, Germany. E-mail: tsabel@uni-goettingen.de

${ }^{2}$ CREST-ENSAE, 3, Avenue Pierre Larousse, 92240 Malakoff,

France.E-mail: Johannes.Schmidt.Hieber@ensae.fr

Abstract In this supplement we give the proofs of Theorem 3 and Corollary 1 and state some technical lemmas.

\section{Appendix B}

\section{B.1 Proof of Theorem 3}

We use the superscript $\downarrow 0$ to indicate the approximation of a function at zero, i.e. define for $\lambda \in(0, \pi]$,

$$
f^{\downarrow 0}(\lambda):=C_{\alpha} \lambda^{2 \alpha} \ell\left(\frac{1}{\lambda}\right), \quad \text { and } \quad \bar{f}^{\downarrow 0}(\lambda):=C_{\alpha} \lambda^{2 \alpha} \ell\left(u_{r_{n}}^{-1}\right),
$$

with $C_{\alpha}=2 \operatorname{sign}(-\alpha) \Gamma(-2 \alpha) \cos (\pi \alpha)$. Similarly

$$
h^{\downarrow 0}(\lambda):=\sigma^{2} f^{\downarrow 0}(\lambda)+\tau^{2} \lambda^{2 K} \quad \text { and } \quad \bar{h}^{\downarrow 0}(\lambda):=\sigma^{2} \bar{f}^{\downarrow 0}(\lambda)+\tau^{2} \lambda^{2 K} .
$$

In order to prove Theorem 3, we will show in the following propositions that up to negligible terms

$$
\int_{0}^{u_{r_{n}^{+}}}\left(\frac{f(\lambda)}{h(\lambda)}\right)^{2} d \lambda=\int_{0}^{u_{n}^{+}}\left(\frac{f^{\downarrow 0}(\lambda)}{h^{\downarrow 0}(\lambda)}\right)^{2} d \lambda=\int_{0}^{u_{r_{n}^{+}}}\left(\frac{\bar{f}^{\downarrow 0}(\lambda)}{\bar{h}^{\downarrow 0}(\lambda)}\right)^{2} d \lambda,
$$

where $\left(r_{n}^{+}\right)_{n}$ is a generic integer sequence satisfying $r_{n} \ll r_{n}^{+} \ll n$.

Proposition B.1. Work under the assumptions of Theorem 2. For any non-negative sequence $\left(\nu_{n}\right)_{n}$, tending to zero,

$$
\int_{0}^{\nu_{n}}\left(\frac{f(\lambda)}{h(\lambda)}\right)^{2} d \lambda=\int_{0}^{\nu_{n}}\left(\frac{f^{\downarrow 0}(\lambda)}{h^{\downarrow 0}(\lambda)}\right)^{2} d \lambda(1+o(1)) .
$$

Proof. By Lemma C.6, $f(\lambda) \sim f^{\downarrow 0}(\lambda)$ for $\lambda \downarrow 0$. Since $\nu_{n} \rightarrow 0$, it follows from (A.6), (A.7), and Taylor expansion of $\sin ^{2 K}(\cdot)$ that

$$
q_{n}:=\sup _{\lambda \in\left(0, \nu_{n}\right]}\left|\frac{f(\lambda)}{f^{\downarrow 0}(\lambda)}-1\right|+\left|\frac{h(\lambda)}{h^{\downarrow 0}(\lambda)}-1\right| \leq \sup _{\lambda \in\left(0, \nu_{n}\right]} 2\left|\frac{f(\lambda)}{f \downarrow 0(\lambda)}-1\right|+\left|\frac{4^{K} \sin ^{2 K}(\lambda / 2)-\lambda^{2 K}}{\lambda^{2 K}}\right|=o(1) .
$$

Therefore, we can estimate

$$
\begin{aligned}
& \left|\int_{0}^{\nu_{n}}\left(\frac{f(\lambda)}{h(\lambda)}\right)^{2}-\int_{0}^{\nu_{n}}\left(\frac{f^{\downarrow 0}(\lambda)}{h(\lambda)}\right)^{2} d \lambda\right| \leq 3 \int_{0}^{\nu_{n}} \frac{\left|f(\lambda)-f^{\downarrow 0}(\lambda)\right|}{(h(\lambda))^{2}} f(\lambda) d \lambda \leq 6 q_{n} \int_{0}^{\nu_{n}}\left(\frac{f(\lambda)}{h(\lambda)}\right)^{2} d \lambda \\
& \quad \text { imsart-bj ver. 2012/08/31 file: Sabe1SchmidtH_Bernoulli_Suppl.tex date: January 31, } 2013
\end{aligned}
$$


and the proof is complete, due to

$$
\begin{aligned}
\left|\int_{0}^{\nu_{n}}\left(\frac{f^{\downarrow 0}(\lambda)}{h(\lambda)}\right)^{2} d \lambda-\int_{0}^{\nu_{n}}\left(\frac{f^{\downarrow 0}(\lambda)}{h^{\downarrow 0}(\lambda)}\right)^{2} d \lambda\right| & \leq 3 \int_{0}^{\nu_{n}}\left|\frac{\left(f^{\downarrow 0}(\lambda)\right)^{2}\left(h^{\downarrow 0}(\lambda)-h(\lambda)\right) h^{\downarrow 0}(\lambda)}{(h(\lambda))^{2}\left(h^{\downarrow 0}(\lambda)\right)^{2}}\right| d \lambda \\
& \leq 12 \int_{0}^{\nu_{n}}\left(\frac{f(\lambda)}{h(\lambda)}\right)^{2}\left|\frac{h(\lambda)}{h^{\downarrow 0}(\lambda)}-1\right| d \lambda \leq 12 q_{n} \int_{0}^{\nu_{n}}\left(\frac{f(\lambda)}{h(\lambda)}\right)^{2} d \lambda .
\end{aligned}
$$

Proposition B.2. Let $\left(r_{n}^{+}\right)_{n}$ be as in (B.3). Then,

$$
\int_{0}^{u_{r_{n}^{+}}}\left(\frac{f^{\downarrow 0}(\lambda)}{h^{\downarrow 0}(\lambda)}\right)^{2} d \lambda=\int_{0}^{u_{r_{n}^{+}}}\left(\frac{\bar{f}^{\downarrow 0}(\lambda)}{\bar{h}^{\downarrow 0}(\lambda)}\right)^{2} d \lambda+o\left(u_{r_{n}} n^{4 \beta}\right) .
$$

Proof. We define $L(\cdot)=(\ell(\cdot))^{2}$, which is again slowly varying. The lemma is proved, once we have established that $|I+I I+I I I+I V|=o\left(u_{r_{n}}\right)$, with

$$
\begin{gathered}
I=\int_{0}^{u_{r_{n}}}\left(\frac{n^{-2 \beta} f^{\downarrow 0}(\lambda)}{h^{\downarrow 0}(\lambda)}\right)^{2} d \lambda-\int_{0}^{u_{r_{n}}}\left(\frac{n^{-2 \beta} \bar{f}^{\downarrow 0}(\lambda)}{h^{\downarrow 0}(\lambda)}\right)^{2} d \lambda, \\
I I=\int_{0}^{u_{r_{n}}}\left(\frac{n^{-2 \beta} \bar{f}^{\downarrow 0}(\lambda)}{h^{\downarrow 0}(\lambda)}\right)^{2} d \lambda-\int_{0}^{u_{r_{n}}}\left(\frac{n^{-2 \beta} \bar{f}^{\downarrow 0}(\lambda)}{\bar{h}^{\downarrow 0}(\lambda)}\right)^{2} d \lambda, \\
I I I=\int_{u_{r_{n}}}^{u_{r_{n}}^{+}}\left(\frac{n^{-2 \beta} f^{\downarrow 0}(\lambda)}{h^{\downarrow 0}(\lambda)}\right)^{2} d \lambda-\int_{u_{r_{n}}}^{u_{r_{n}^{+}}}\left(\frac{n^{-2 \beta} \bar{f}^{\downarrow 0}(\lambda)}{h^{\downarrow 0}(\lambda)}\right)^{2} d \lambda, \\
I V=\int_{u_{r_{n}}}^{u_{r_{n}^{+}}}\left(\frac{n^{-2 \beta} \bar{f}^{\downarrow 0}(\lambda)}{h^{\downarrow 0}(\lambda)}\right)^{2} d \lambda-\int_{u_{r_{n}}}^{u_{r_{n}^{+}}}\left(\frac{n^{-2 \beta} \bar{f}^{\downarrow 0}(\lambda)}{\bar{h}^{\downarrow 0}(\lambda)}\right)^{2} d \lambda .
\end{gathered}
$$

(I): Note that by substituting $s=u_{r_{n}} \lambda^{-1}$,

$$
|I| \leq \sigma^{-4} \int_{0}^{u_{r_{n}}} \frac{\left|L(1 / \lambda)-L\left(u_{r_{n}}^{-1}\right)\right|}{L(1 / \lambda)} d \lambda=u_{r_{n}} \sigma^{-4} \int_{1}^{\infty} \frac{\left|L\left(s u_{r_{n}}^{-1}\right)-L\left(u_{r_{n}}^{-1}\right)\right|}{L\left(s u_{r_{n}}^{-1}\right)} s^{-2} d s .
$$

Since $u_{r_{n}}^{-1} \rightarrow \infty$, we find by Potter's bound that $\left|L\left(u_{r_{n}}^{-1}\right) / L\left(s u_{r_{n}}^{-1}\right)\right| \leq 2 s^{1 / 2}$, for sufficiently large $n$ and all $s \in[1, \infty)$. Therefore, by dominated convergence and the definition of slowly varying functions,

$$
\lim _{n \rightarrow \infty} \int_{1}^{\infty} \frac{\left|L\left(s u_{r_{n}}^{-1}\right)-L\left(u_{r_{n}}^{-1}\right)\right|}{L\left(s u_{r_{n}}^{-1}\right)} s^{-2} d s=\int_{1}^{\infty} \lim _{n \rightarrow \infty} \frac{\left|L\left(s u_{r_{n}}^{-1}\right)-L\left(u_{r_{n}}^{-1}\right)\right|}{L\left(s u_{r_{n}}^{-1}\right)} s^{-2} d s=0
$$

and therefore $I=o\left(u_{r_{n}}\right)$.

(II): Let $0<a_{1}, a_{2}, b$. Then,

$$
\left|\frac{1}{\left(a_{1}+b\right)^{2}}-\frac{1}{\left(a_{2}+b\right)^{2}}\right|=\left(\frac{1}{a_{1}+b}+\frac{1}{a_{2}+b}\right) \frac{\left|a_{1}-a_{2}\right|}{\left(a_{1}+b\right)\left(a_{2}+b\right)} \leq\left(\frac{1}{a_{1}}+\frac{1}{a_{2}}\right) \frac{\left|a_{1}-a_{2}\right|}{a_{1} a_{2}}=\left|\frac{1}{a_{1}^{2}}-\frac{1}{a_{2}^{2}}\right| .
$$

With $a_{1}=\sigma^{2} n^{-2 \beta} \bar{f}^{\downarrow 0}(\lambda), a_{2}=\sigma^{2} n^{-2 \beta} f^{\downarrow 0}(\lambda)$ and $b=h^{\downarrow 0}(\lambda)-a_{2}$,

$$
|I I| \leq \sigma^{-4} \int_{0}^{u_{r_{n}}}\left|\frac{\left(\bar{f}^{\downarrow 0}(\lambda)\right)^{2}}{\left(f^{\downarrow 0}(\lambda)\right)^{2}}-1\right| d \lambda=\sigma^{-4} \int_{0}^{u_{r_{n}}} \frac{\left|L(1 / \lambda)-L\left(u_{r_{n}}^{-1}\right)\right|}{L(1 / \lambda)} d \lambda=o\left(u_{r_{n}}\right),
$$

where the last step follows from $(I)$.

(III): By estimating $h^{\downarrow 0}(\lambda) \geq \tau^{2} \lambda^{2 K}$,

$$
\begin{aligned}
|I I I| & \leq \int_{u_{r_{n}}}^{u_{r_{n}^{+}}}\left(C \tau^{-2} n^{-2 \beta} \lambda^{2 \alpha-2 K}\right)^{2}\left|L(1 / \lambda)-L\left(u_{r_{n}}^{-1}\right)\right| d \lambda \\
& =C^{2} \tau^{-4} n^{-4 \beta} u_{r_{n}}^{1+4 \alpha-4 K} L\left(u_{r_{n}}^{-1}\right) \int_{1}^{u_{r_{n}^{+}} / u_{r_{n}}} \lambda^{4 \alpha-4 K}\left|\frac{L\left(u_{r_{n}}^{-1} / \lambda\right)-L\left(u_{r_{n}}^{-1}\right)}{L\left(u_{r_{n}}^{-1}\right)}\right| d \lambda \\
& \lesssim u_{r_{n}} L\left(u_{r_{n}}^{-1}\right)\left(L\left(n^{\frac{\beta}{K-\alpha}}\right)\right)^{-1} \int_{1}^{u_{r_{n}^{+}} / u_{r_{n}}} \lambda^{4 \alpha-4 K}\left|\frac{L\left(u_{r_{n}}^{-1} / \lambda\right)-L\left(u_{r_{n}}^{-1}\right)}{L\left(u_{r_{n}}^{-1}\right)}\right| d \lambda .
\end{aligned}
$$


By arguing similar as in $(I)$ and $(I I)$, and since $4 \alpha-4 K<-1$, we see that the integral tends to zero and therefore, using Lemma A.2, $|I I I|=o\left(u_{r_{n}}\right)$ follows.

$(I V)$ : Using $\left(\bar{h}^{\downarrow 0}(\lambda)\right)^{-1}+\left(h^{\downarrow 0}(\lambda)\right)^{-1} \leq \sigma^{-2} n^{2 \beta}\left(\bar{f}^{\downarrow 0}(\lambda)\right)^{-1}+\sigma^{-2} n^{2 \beta}\left(f^{\downarrow 0}(\lambda)\right)^{-1}$ and the elementary inequality $(a b)^{-1} \leq a^{-2}+b^{-2}$ for $a, b>0$, we obtain

$$
\begin{aligned}
|I V| & \leq \sigma^{-2} n^{2 \beta} \int_{u_{r_{n}}}^{u_{r_{n}^{+}}}\left(\bar{f}^{\downarrow 0}(\lambda)\right)^{2}\left(\frac{1}{\bar{f}^{\downarrow 0}(\lambda)}+\frac{1}{f^{\downarrow 0}(\lambda)}\right) \frac{\left|f^{\downarrow 0}(\lambda)-\bar{f}^{\downarrow 0}(\lambda)\right|}{\bar{h}^{\downarrow 0}(\lambda) h^{\downarrow 0}(\lambda)} d \lambda \\
& \leq \sigma^{-2} n^{2 \beta} \int_{u_{r_{n}}}^{u_{r_{n}^{+}}} \frac{\left(\bar{f}^{\downarrow 0}(\lambda)\right)^{2}}{\bar{h}^{\downarrow 0}(\lambda) h \downarrow 0(\lambda)}\left|\left(f^{\downarrow 0}(\lambda)\right)^{2}-\left(\bar{f}^{\downarrow 0}(\lambda)\right)^{2}\right|\left(\left(f^{\downarrow 0}(\lambda)\right)^{-2}+\left(\bar{f}^{\downarrow 0}(\lambda)\right)^{-2}\right) d \lambda .
\end{aligned}
$$

By expanding the last sum and treating each of the two terms separately, we can argue along the lines of $(I I I)$.

Proof of Theorem 3. We consider two cases. First assume that $K-\alpha>1 / 4$. Let $\left(r_{n}^{+}\right)_{n}$ be an integer sequence such that $r_{n}^{+} \ll r_{n}$. Define

$$
C_{n}:=n^{-2 \beta} \ell\left(u_{r_{n}}^{-1}\right) 2 \Gamma(-2 \alpha) \cos (\pi \alpha) \quad \text { and } \quad C_{n}^{\prime}:=\left(C_{n}^{-1} \sigma^{-2} \tau^{2}\right)^{1 /(2 K-2 \alpha)} .
$$

Application of Propositions C.1, B.1, and B.2 and substitution show that

$$
\begin{aligned}
\int_{0}^{\pi} \frac{f^{2}(\lambda)}{h^{2}(\lambda)} d \lambda & =\sigma^{-4} n^{4 \beta} \int_{0}^{u_{r_{n}^{+}}}\left(1+C_{n}^{-1} \sigma^{-2} \tau^{2} \lambda^{2 K-2 \alpha}\right)^{-2} d \lambda(1+o(1))+o\left(r_{n} n^{4 \beta}\right) \\
& =\left(C_{n}^{\prime}\right)^{-1} \sigma^{-4} n^{4 \beta} \int_{0}^{u_{r_{n}^{+}} / C_{n}^{\prime}}\left(1+\lambda^{2 K-2 \alpha}\right)^{-2} d \lambda(1+o(1))+o\left(r_{n} n^{4 \beta}\right) .
\end{aligned}
$$

Observe that by Lemma A.2, $u_{r_{n}^{+}} / C_{n}^{\prime} \rightarrow \infty$. Since $K-\alpha>1 / 4$ the integral $\int_{0}^{\infty}\left(1+\lambda^{2 K-2 \alpha}\right)^{-2} d \lambda$ is finite and we can approximate the r.h.s. of (B.4) further by $\int_{0}^{\infty}\left(1+\lambda^{2 K-2 \alpha}\right)^{-2} d \lambda(1+o(1))$. By using formula (3.251.6) in Gradshteyn and Ryzhik [28],

$$
\int_{0}^{\infty}\left(\frac{1}{1+\lambda^{2 K-2 \alpha}}\right)^{2} d \lambda=\frac{1}{K-\alpha} \int_{0}^{\infty} \frac{\lambda^{\frac{1}{K-\alpha}}-1}{\left(1+\lambda^{2}\right)^{2}} d \lambda=\frac{\pi\left(\frac{1}{K-\alpha}-2\right)}{4(K-\alpha) \sin \left(\pi\left(\frac{1}{K-\alpha}-2\right) / 2\right)}
$$

and for $K-\alpha=1 / 2$ the r.h.s. is defined by 1 . Combining the last identities (recall that for the Fisher information, the integrals are scaled by $\left.n^{1-4 \beta} /(2 \pi)\right)$ and application of Lemma A.2 completes the proof for the first case.

Now, let us prove the second statement of Theorem 3, i.e. the case $K-\alpha<1 / 4$, i.e. $K=0$ and $\alpha \in(-1 / 4,-\beta)$. With the upper bound in (A.6) (extended for all $\lambda \in(0, \pi])$, we have $f(\lambda) \lesssim \lambda^{2 \alpha-\epsilon}$ and $\int_{0}^{n^{\beta / \alpha}} f^{2}(\lambda)\left(h^{-2}(\lambda)+1\right) d \lambda=o(1)$. Since, $1 / h-\tau^{-2}=\sigma^{2} \tau^{-2} n^{-2 \beta} f / h$ we obtain also

$$
\int_{n^{\frac{\beta}{\alpha}}}^{\pi}\left|\frac{f^{2}(\lambda)}{h^{2}(\lambda)}-\tau^{-4} f^{2}(\lambda)\right| d \lambda \leq 2 \sigma^{2} \tau^{-6} n^{-2 \beta} \int_{n^{\frac{\beta}{\alpha}}}^{\pi} f^{3}(\lambda) d \lambda \lesssim n^{-2 \beta} \int_{n^{\frac{\beta}{\alpha}}}^{\pi} \lambda^{6 \alpha-3 \epsilon} d \lambda=o(1)
$$

for small $\epsilon$. Combining the last approximations yields

$$
I_{\sigma^{2}}^{n}=\frac{n^{1-4 \beta}}{2 \pi \tau^{4}} \int_{0}^{\pi} f^{2}(\lambda) d \lambda+o\left(n^{1-4 \beta}\right)=\frac{n^{1-4 \beta}}{2 \tau^{4}} \sum_{k=-\infty}^{\infty} \gamma_{k}^{2}+o\left(n^{1-4 \beta}\right)
$$

since $f=\sum_{k=-\infty}^{\infty} \cos (k \cdot) \gamma_{k}$. This completes the proof of $(2.14)$.

Finally, we evaluate the integral in the critical case, i.e. we prove $(2.15)$. Because $\log (x) \ell^{2}(x) \rightarrow$ $\infty$ for $x \rightarrow \infty$, one can find a non-negative sequence $\left(\nu_{n}\right)_{n}$, tending to zero, such that

$$
\int_{\nu_{n}}^{\pi}\left(\frac{f(\lambda)}{h(\lambda)}\right)^{2} d \lambda \lesssim \int_{\nu_{n}}^{\pi} \lambda^{-1-2 \epsilon} d \lambda \lesssim \nu_{n}^{-2 \epsilon}=o\left(\log (n) \ell^{2}\left(n^{4 \beta}\right)\right)
$$


where we used the global upper bound for $f$, cf. (A.6), in the first inequality. Since $\nu_{n}$ tends to zero, Proposition B.1 applies. With $f^{\downarrow 0}$ and $h^{\downarrow 0}$ as defined in (B.1) and (B.2),

$$
\begin{aligned}
I_{\sigma^{2}}^{n} & =\frac{n^{1-4 \beta}}{2 \pi} \int_{0}^{\nu_{n}}\left(\frac{f^{\downarrow 0}(\lambda)}{h^{\downarrow 0}(\lambda)}\right)^{2} d \lambda(1+o(1))+o\left(n^{1-4 \beta} \log (n) \ell^{2}\left(n^{4 \beta}\right)\right) \\
& =\frac{n^{1-4 \beta}}{2 \pi} \int_{q_{n}}^{\nu_{n}}\left(\frac{f^{\downarrow 0}(\lambda)}{h^{\downarrow 0}(\lambda)}\right)^{2} d \lambda(1+o(1))+o\left(n^{1-4 \beta} \log (n) \ell^{2}\left(n^{4 \beta}\right)\right) .
\end{aligned}
$$

Note that $K-\alpha=1 / 4$ implies $K=0$ and $\alpha=-1 / 4$. Observe also that by Potter's bound,

$$
\begin{aligned}
\int_{q_{n}}^{\nu_{n}}\left(f^{\downarrow 0}(\lambda)\right)^{2}\left|\left(h^{\downarrow 0}(\lambda)\right)^{-2}-\tau^{-4}\right| d \lambda & \lesssim n^{-2 \beta} \int_{q_{n}}^{\nu_{n}}\left(f^{\downarrow 0}(\lambda)\right)^{3} d \lambda \\
& =n^{-2 \beta} \ell^{3}\left(q_{n}^{-1}\right) q_{n}^{-1 / 2} \int_{1}^{\nu_{n} / q_{n}} \lambda^{-3 / 2} \frac{\ell^{3}\left(\frac{1}{\lambda q_{n}}\right)}{\ell^{3}\left(\frac{1}{q_{n}}\right)} d \lambda=O\left(\ell^{2}\left(n^{4 \beta}\right)\right) .
\end{aligned}
$$

For $\alpha=-1 / 4, f^{\downarrow 0}(\lambda)=\sqrt{2 \pi} \lambda^{-1 / 2} \ell(1 / \lambda)$. This shows

$$
I_{\sigma^{2}}^{n}=n^{1-4 \beta} \tau^{-4} \int_{q_{n}}^{1} \ell^{2}\left(\frac{1}{\lambda}\right) \frac{d \lambda}{\lambda}(1+o(1))+o\left(n^{1-4 \beta} \log (n) \ell^{2}\left(n^{4 \beta}\right)\right),
$$

where the integral $\int_{\nu_{n}}^{1}$ is estimated as in (B.5).

\section{B.2 Proof of Corollary 1}

Proof. For $H=1 / 2$ the result follows from [14]. If $H \neq 1 / 2$, we check the conditions of Theorem 3 (i.e. Theorem 2). Note that the three versions of Theorem 2 correspond to Hurst indices $0<$ $H<1 / 2,1 / 2<H<3 / 4$, and $3 / 4 \leq H<1$, respectively. By using the properties of fGN, listed in Lemma C.7 (for instance in order to show $\sum_{k=-\infty}^{\infty} \gamma_{k}=0$ for $0<H<1 / 2$, use Lemma C.7 (i)), part (1) and part (2) of Theorem 2 are obviously fulfilled, but some work has to be done for the third part.

To bound the increments of the autocovariance function, i.e. $\left|\gamma_{p+1}-\gamma_{p}\right|=\left|r_{H}(p+1)-r_{H}(p)\right| \lesssim$ $r_{H}(p) / p=\gamma_{p} / p$, define on $\mathbb{R}^{+}, x \mapsto g(x)=|x|^{2 H}$. Then there exists $\xi_{1} \in[x, x+1]$ and $\xi_{2} \in[x-1, x]$ such that by Taylor expansion $g(x+1)=\sum_{k=0}^{3}(k !)^{-1} g^{(k)}(x)+24^{-1} g^{(4)}\left(\xi_{1}\right)$ and $g(x-1)=$ $\sum_{k=0}^{3}(-1)^{k}(k !)^{-1} g^{(k)}(x)+24^{-1} g^{(4)}\left(\xi_{2}\right)$. Thus,

$$
r_{H}(q)=g^{\prime \prime}(q)+\frac{1}{24}\left(g^{(4)}\left(\xi_{1}\right)+g^{(4)}\left(\xi_{2}\right)\right) .
$$

By another Taylor expansion and $p \geq 1,\left|r_{H}(p+1)-r_{H}(p)\right| \lesssim p^{2 H-3}$.

\section{Appendix C Further technicalities}

First, let us summarize some facts about Frobenius norms.

Lemma C.1. Given $n \times n$ matrices $A=\left(a_{i, j}\right)_{i, j=1, \ldots, n}, B, C$. Let $\langle A\rangle=\operatorname{tr}\left(A^{2}\right)$ and denote by $\|A\|_{2}=\left(\sum_{i, j} a_{i, j}^{2}\right)^{1 / 2}$ the Frobenius (or Hilbert-Schmidt) norm. Then,

(i) $\langle A\rangle \leq\|A\|_{2}^{2}$, and equality holds if $A$ is symmetric.

(ii) $A$ and $B$ are circular commuting, i.e. $\langle A B\rangle=\langle B A\rangle$

(iii) If $A \geq 0$ and $B$ Hermitian, then, $\langle A B\rangle \leq\|A\|_{\infty}^{2}\langle B\rangle$.

(iv) If $C \geq 0$ as well as $A$ and $B$ Hermitian, then

$$
\langle C(A+B)\rangle=\left\|C^{1 / 2}(A+B) C^{1 / 2}\right\|_{2}^{2} \leq 2\langle C A\rangle+2\langle C B\rangle .
$$


Proof. (i): Due to $2 u v \leq u^{2}+v^{2}, \operatorname{tr}\left(A^{2}\right)=\left(\sum_{i, j} a_{i, j} a_{j, i}\right)^{2} \leq \sum_{i, j} a_{i, j}^{2}=\|A\|_{2}^{2}$. (iii): Write $A=D^{t} \Lambda D$ with $\Lambda$ diagonal.

The next result is well-known, cf. Sánchez et al. [29], p. 2635. For sake of completeness we give a short proof.

Lemma C.2. Assume that $g=\sum_{m=-n}^{n} g_{m} \cos (m \cdot)$ with $g_{m}=g_{-m}$ and let $D_{n}(g)$ be defined as in (A.4). Then, we have for the $(i, j)$-th entry $\left(D_{n}(g)\right)_{i, j}=g_{|i-j|}+g_{i+j-1}$ if $i+j \leq n+1$ and $\left(D_{n}(g)\right)_{i, j}=g_{|i-j|}-g_{2 n+2-i-j}$ if $i+j>n+1$.

Proof. Recall (A.2) and (A.4). Expand

$$
\begin{array}{r}
\left(D_{n}(g)\right)_{i j}=\frac{1}{2 n+1} \sum_{m=-n}^{n} g_{m} \sum_{k=1}^{n}\left[\cos \left((i+j-1+m) u_{k}\right)+\cos \left((i+j-1-m) u_{k}\right)\right. \\
\left.+\cos \left((i-j+m) u_{k}\right)+\cos \left((i-j-m) u_{k}\right)\right]
\end{array}
$$

using $g\left(u_{k}\right)=\sum_{m=-n}^{n} g_{m} \cos \left(m u_{k}\right)$ and $2 \cos (x) \cos (y)=\cos (x+y)+\cos (x-y)$. With the wellknown summation formula for Dirichlet kernels, we find that for an integer $\ell, \sum_{k=1}^{n} \cos \left(\ell u_{k}\right)$ equals $n(-1)^{\ell /(2 n+1)}$, if $\ell$ is divisible by $2 n+1$, and $\frac{1}{2}(-1)^{\ell+1}$ otherwise. If we rewrite in the first case $n(-1)^{\ell /(2 n+1)}$ as $\left(n+\frac{1}{2}\right)(-1)^{\ell /(2 n+1)}+\frac{1}{2}(-1)^{\ell+1}$, we find that $\left(D_{n}(g)\right)_{i, j}=g_{|i-j|}+g_{i+j-1}$ if $i+j \leq n+1$ and $\left(D_{n}(g)\right)_{i, j}=g_{|i-j|}-g_{2 n+2-i-j}$ if $i+j>n+1$.

In the next lemma, we collect a number of properties of general monotone sequences.

Lemma C.3. Assume that $\left(a_{k}\right)_{k} \in \mathrm{GM}$ and define $f=a_{0}+2 \sum_{k=1}^{\infty} a_{k} \cos (k \cdot)$.

(i) If $\sum_{k=1}^{\infty} k^{-1}\left|a_{k}\right|<\infty$, then there exists a constant $C$ such that

$$
\left|f(x)-S_{n} f(x)\right| \leq C \frac{1}{x}\left(\left|a_{n}\right|+\sum_{k=n+1}^{\infty} \frac{\left|a_{k}\right|}{k}\right), \quad \text { if } x \in\left[\frac{1}{n}, \pi\right] .
$$

(ii) If additionally $a_{k} \sim k^{-2 \alpha-1} \ell(k)$ holds for $\alpha>-1 / 2$ and a slowly varying function $\ell$, then $\left(a_{k}\right)_{k}$ is of bounded variation, i.e. $\sum_{k=1}^{\infty}\left|a_{k+1}-a_{k}\right|<\infty$.

(iii) If $a_{k} \sim k^{-2 \alpha-1} \ell(k)$ holds for $\alpha \in(-1 / 2,0)$ and a quasi-monotone slowly varying function $\ell$, then

$$
f(\lambda) \sim 2 \lambda^{2 \alpha} \ell\left(\frac{1}{\lambda}\right) \Gamma(-2 \alpha) \cos (\pi \alpha)
$$

Proof. ( $i$ ) and (iii) can be found in the recent paper by Tikhonov [24]. To verify (ii) note that for sufficiently large $K$, using the definition of GM and the same bound as in (A.5), $\sum_{k=K}^{\infty}\left|a_{k+1}-a_{k}\right| \leq$ $C \sum_{q=0}^{\infty}\left|a_{2^{q} K}\right|<\infty$.

The following lemma gives a very useful bound on the Frobenius norm between the matrices $T_{n}(f)$ and $D_{n}(f)$ defined in (A.3) and (A.4).

Lemma C.4. Let $f=\sum_{k=0}^{\infty} a_{k} \cos (k \cdot)$ and assume that $\left(a_{k}\right) \in \mathrm{GM}$. If $\sum_{k \geq 1} k^{-1}\left|a_{k}\right|<\infty$, then, there exists a constant $C$, such that

$$
\left\|D_{n}(f)-T_{n}(f)\right\|_{2} \leq C n\left(\left|a_{n}\right|+\sum_{k=n+1}^{\infty} \frac{\left|a_{k}\right|}{k}\right)+\left(2 \sum_{k=1}^{n} k a_{k}^{2}\right)^{1 / 2} .
$$


Proof. Note that $\left\|D_{n}(f)-T_{n}(f)\right\|_{2} \leq\left\|D_{n}(f)-D_{n}\left(S_{n} f\right)\right\|_{2}+\left\|D_{n}\left(S_{n} f\right)-T_{n}(f)\right\|_{2}$. In order to bound the first part, we use the estimate from Lemma C.3 (i) together with (A.4) and hence $\left\|D_{n}(f)-D_{n}\left(S_{n} f\right)\right\|_{2}^{2}=\sum_{k=1}^{n}\left(f\left(u_{k}\right)-S_{n} f\left(u_{k}\right)\right)^{2}$ for $u_{k}$ as in (A.2). Since $u_{k} \geq 1 / n$, this yields

$$
\left\|D_{n}(f)-D_{n}\left(S_{n} f\right)\right\|_{2} \leq C_{1} n\left(\left|a_{n}\right|+\sum_{k=n+1}^{\infty} \frac{\left|a_{k}\right|}{k}\right)
$$

for another constant $C_{1}$. Using Lemma C.2 and $\left(T_{n}(f)\right)_{i, j}=f_{|i-j|}$, the second term can be bounded easily by $\left(2 \sum_{k=1}^{n} k f_{k}^{2}\right)^{1 / 2}$.

Lemma C.5. Assume that $\left(\gamma_{k}\right)_{k}$ satisfies the assumptions of Theorem 2, Version 3. Then it is also in GM.

Proof. The following arguments are for $J$ large enough. Observe that by Potter's bound, the sequence $\left(b_{J}\right)_{J}$,

$$
b_{J}:=\frac{1}{J} \sum_{j=J}^{2 J-1} \frac{\ell(j)}{\ell(J)}\left(\frac{j}{J}\right)^{-2 \alpha-2}
$$

is uniformly bounded. Using also (A.5)

$$
\sum_{j=J}^{2 J-1}\left|\gamma_{j+1}-\gamma_{j}\right| \leq C_{1} \sum_{j=J}^{2 J-1} \frac{\gamma_{j}}{j} \lesssim C_{1} b_{J} \ell(J) J^{-2 \alpha-1} \lesssim \gamma_{J} .
$$

Lemma C.6. Under the assumptions of Theorem 2, we have that $f$ is bounded on any interval $[\delta, \pi]$ with $\delta>0$ and

$$
f(\lambda) \sim 2 \operatorname{sign}(-\alpha) \Gamma(-2 \alpha) \cos (\pi \alpha) \lambda^{2 \alpha} \ell(1 / \lambda) .
$$

Proof. For $\alpha \in(0,1 / 2)$, the sequence $\left(\gamma_{k}\right)_{k}$ is absolutely summable, and hence $f$ is bounded on $[0, \pi]$. The behavior at zero, i.e. $f(\lambda) \sim-2 \Gamma(-2 \alpha) \cos (\pi \alpha) \lambda^{2 \alpha} \ell(1 / \lambda)$, follows from Theorem 1.2 in [27] and the assumption $\sum_{k=-\infty}^{\infty} \gamma_{k}=0$. For $\alpha \in(-1 / 2,0)$, we have $\left(\gamma_{k}\right)_{k} \in$ GM (cf. Lemma C.5). Hence, by Lemma C.3 (ii), $\left(\gamma_{k}\right)_{k}$ is of bounded variation and therefore (cf. Zygmund [30], Theorem 2.6) $f$ is bounded on $[\delta, \pi]$ for every $\delta>0$. The second property is a consequence of Lemma C.3 (iii) and Assumption 1.

Proposition C.1. Work under the assumptions of Theorem 4 with $K-\alpha>1 / 4$ and let $\left(r_{n}^{+}\right)_{n}$ be an integer sequence satisfying $r_{n} \ll r_{n}^{+} \ll n$. Then,

$$
n \int_{u_{r_{n}^{+}}}^{\pi}\left(\frac{f(\lambda)}{h(\lambda)}\right)^{2} d \lambda=o\left(r_{n} n^{4 \beta}\right) \quad \text { and } \sum_{i=r_{n}^{+}+1}^{n}\left(\frac{f\left(u_{i}\right)}{h\left(u_{i}\right)}\right)^{2}=o\left(r_{n} n^{4 \beta}\right) .
$$

Proof. Because of $K-\alpha>1 / 4$, there exist $\delta^{\prime}>0$ and an integer $n_{0}$ such that by Potter's bound we find for all $n$ larger than $n_{0}$,

$$
\frac{\ell\left(\frac{1}{\mu u_{r_{n}^{+}}}\right)}{\ell\left(\frac{1}{u_{r_{n}^{+}}}\right)} \leq 2 \mu^{K-\alpha-1 / 4}, \text { for all } \mu \in\left(0, \delta^{\prime} / u_{r_{n}^{+}}\right] .
$$

Let $\delta$ be as in (A.6). Together with (A.7), we obtain

$$
\begin{aligned}
\frac{f\left(u_{r_{n}^{+}} \mu\right)}{h\left(u_{r_{n}^{+}} \mu\right)} & \leq 2 \cdot 4^{K} \tau^{-2} u_{r_{n}^{+}}^{2 \alpha-2 K} \mu^{2 \alpha-2 K} \ell\left(\frac{1}{\mu u_{r_{n}^{+}}}\right) \\
& \leq 4^{K+1} \tau^{-2} u_{r_{n}^{+}}^{2 \alpha-2 K} \mu^{\alpha-K-1 / 4} \ell\left(\frac{1}{u_{r_{n}^{+}}}\right), \quad \text { for all } \mu \in\left(0,\left(\delta \wedge \delta^{\prime}\right) / u_{r_{n}^{+}}\right] .
\end{aligned}
$$


Since $f$ is bounded on $\left[\delta \wedge \delta^{\prime}, \pi\right]$ under the assumptions of Theorem 2 (cf. Lemma C.6), we also have $f(\lambda) / h(\lambda) \lesssim \lambda^{-2 K}$, for all $\lambda \in\left(\delta \wedge \delta^{\prime}, \pi\right]$. Now, we are ready to prove the two statements (denoted by $(I)$ and $(I I))$ of the proposition.

$(I)$ : Decomposing $\int_{u_{r_{n}^{+}}}^{\pi}=\int_{u_{r_{n}^{+}}}^{\delta \wedge \delta^{\prime}}+\int_{\delta \wedge \delta^{\prime}}^{\pi}$ and substituting $\mu=\lambda / u_{r_{n}^{+}}$in the first integral yields

$$
\begin{aligned}
\int_{u_{r_{n}^{+}}}^{\pi}\left(\frac{f(\lambda)}{h(\lambda)}\right)^{2} d \lambda & =u_{r_{n}^{+}} \int_{1}^{\left(\delta \wedge \delta^{\prime}\right) / u_{r_{n}^{+}}}\left(\frac{f\left(u_{r_{n}^{+}} \mu\right)}{h\left(u_{r_{n}^{+}} \mu\right)}\right)^{2} d \mu+\int_{\delta \wedge \delta^{\prime}}^{\pi}\left(\frac{f(\lambda)}{h(\lambda)}\right)^{2} d \lambda \\
& \leq 4^{2 K+2} \tau^{-4} u_{r_{n}^{+}}^{4 \alpha-4 K+1} \ell^{2}\left(\frac{1}{u_{r_{n}^{+}}}\right) \int_{1}^{\infty} \mu^{2 \alpha-2 K-1 / 2} d \mu+o\left(u_{r_{n}} n^{4 \beta}\right) .
\end{aligned}
$$

Note that for two positive sequences $\left(a_{n}\right)_{n}$ and $\left(b_{n}\right)_{n}$ with $a_{n} \ll b_{n}$, and any $\kappa>0$, we have for sufficiently large $n$,

$$
\frac{\ell\left(a_{n}\right)}{\ell\left(b_{n}\right)} \leq 2\left(\frac{b_{n}}{a_{n}}\right)^{\kappa / 2} \ll\left(\frac{b_{n}}{a_{n}}\right)^{\kappa} .
$$

Apply this for $a_{n}=1 / u_{r_{n}^{+}}$and $b_{n}=1 / u_{r_{n}}$ together with Lemma A.2. Then,

$$
u_{r_{n}^{+}}^{4(\alpha-K)+1} \ell^{2}\left(\frac{1}{u_{r_{n}^{+}}}\right) \ll u_{r_{n}}^{4(\alpha-K)+1} \ell^{2}\left(\frac{1}{u_{r_{n}}}\right)=O\left(u_{r_{n}} n^{4 \beta}\right)
$$

and this completes the proof of $(I)$.

(II): Let $\kappa_{n}$ be the largest integer, s.t. $u_{\kappa_{n}} \leq \delta \wedge \delta^{\prime}$. Similarly as for $(I)$, we rewrite

$$
\sum_{i=r_{n}^{+}+1}^{n}\left(\frac{f\left(u_{i}\right)}{h\left(u_{i}\right)}\right)^{2} \leq 4^{2 K+2} \tau^{-4} u_{r_{n}^{+}}^{4 \alpha-4 K} \ell^{2}\left(\frac{1}{u_{r_{n}^{+}}}\right) \sum_{i=r_{n}^{+}+1}^{\kappa_{n}}\left(\frac{u_{i}}{u_{r_{n}^{+}}}\right)^{2 \alpha-2 K-1 / 2}+o\left(r_{n} n^{4 \beta}\right) .
$$

Note that

$$
\frac{1}{r_{n}^{+}} \sum_{i=r_{n}^{+}+1}^{\kappa_{n}}\left(\frac{u_{i}}{u_{r_{n}^{+}}}\right)^{2 \alpha-2 K-1 / 2} \leq \sum_{i=r_{n}^{+}+1}^{\infty}\left(\frac{2 i}{r_{n}^{+}}\right)^{2 \alpha-2 K-1 / 2} \leq \int_{1}^{\infty}(2 \lambda)^{2 \alpha-2 K-1 / 2} d \lambda<\infty .
$$

Arguing as above yields the required result.

Finally, we summarizes a number of facts on fractional Gaussian noise.

Lemma C.7. Let $\left(N_{k}^{H}\right)_{k \in \mathbb{N}}$ denote a fractional Gaussian noise process, i.e. a centered Gaussian process with covariance

$$
\operatorname{Cov}\left(N_{k}^{H}, N_{j}^{H}\right)=\frac{1}{2}\left(|k-j+1|^{2 H}-2|k-j|^{2 H}+|k-j-1|^{2 H}\right) .
$$

Denote by $f_{H}$ and $r_{H}$ the spectral density and the autocovariance function, respectively. Then,

(i) $r_{H}(k)=\frac{1}{2}\left(|k+1|^{2 H}-2|k|^{2 H}+|k-1|^{2 H}\right) \sim H(2 H-1) k^{2 H-2}$.

(ii) $\left(r_{H}(k)\right)_{k \in \mathbb{Z}}$ is O-regularly varying quasi-monotone and (therefore also) in $\mathrm{GM}$

(iii) $f_{H}(\lambda)=2 \sin (\pi H) \Gamma(2 H+1)(1-\cos \lambda) \sum_{j=-\infty}^{\infty}|\lambda+2 \pi j|^{-2 H-1} \sim \sin (\pi H) \Gamma(2 H+1) \lambda^{1-2 H}$

(iv) There exist positive constants $C_{H}$ and $C_{H}^{\prime}$, such that $C_{H} \lambda^{1-2 H} \leq f_{H}(\lambda) \leq C_{H}^{\prime} \lambda^{1-2 H}$ for all $\lambda \in(0, \pi]$.

Proof. (i), (iii) and (iv) are well-known (cf. [26]). (ii) follows from the monotone decrease of $\left(r_{H}(k)\right)_{k}$. 


\section{References}

[26] J. Beran. Statistics for Long-Memory Processes. Chapman \& Hall, New York, 1994.

[27] C.-P. Chen and L. Chen. Asymptotic behavior of trigonometric series with O-regularly varying quasimonotone coefficients. J. Math. Anal. Appl., 250:13-26, 2000.

[28] I. S. Gradshteyn and I. M. Ryzhik. Table of Integrals, Series, and Products. Elsevier Academic Press, Burlington, 2007.

[29] V. Sánchez, P. García, A. Peinado, J. Segura, and A. Rubio. Diagonalizing properties of the discrete cosine transform. IEEE Trans. Signal Process., 43:2631-2641, 1995.

[30] A. Zygmund. Trigonometric Series. Cambridge University Press, Cambridge, 2002. 